\title{
Genetic Diversity in Appemidi (Unique Aromatic Pickle Mango) Genotypes from Western Ghats Regions of Chikmagalur (Karnataka), India
}

\author{
G.L. Veena* and M.R. Dinesh \\ Division of Fruit Corps, ICAR-IIHR, Bengaluru-560089 \\ Director, ICAR-IIHR, Bengaluru-560089, India \\ *Corresponding author
}

\section{A B S T R A C T}

\begin{tabular}{|l|}
\hline K e y w o r d s \\
Genetic diversity, \\
Appemidi, Western \\
ghats regions, \\
Breeding programme \\
\hline Article Info \\
\hline $\begin{array}{l}\text { Accepted: } \\
\text { 08 June } 2018 \\
\text { Available Online: } \\
\text { 10 July } 2018\end{array}$ \\
\hline
\end{tabular}

Keywords

Genetic diversity, ghats regions,

Article Info

Accepted:

Available Online:

\section{Introduction}

India is a land of diversity with diverse flora and fauna. Mango the most popular and choicest fruit crop of India having originated in Indo-Myanmar region (Mukherjee, 1953), both wild and cultivated forms of mango exhibit unusual diversity in fruit forms, flavour and taste (Mukherjee, 1948; Naik and Gangolly, 1950). Western Ghats and the peninsular region of India are diversity rich centres for Mangifera indica varieties. There are several unique types which are highly aromatic and unique taste for pickling which is commonly known as Appemidi found in
Western Ghats regions of Karnataka. A very large unexploited varietal diversity in mango can be seen especially in the Western Ghats region of Karnataka. This region is known for the pickling types of mango viz., 'Appemidi' (midi means tender mango in Kannada). These are found on the river banks, which is also one of the reasons for this diversity. The appemidi is not just an ordinary mango; its fragrance is so strong that adding just a few midis to an ordinary pickle can change its taste and aroma. "Appemidi" is the king of all tender mangoes as far as its use in pickle industry is concerned. The local populace who has conserved few of them in their backyard after 
evaluating them for varied uses has selected some of these. Since, there is a large unexplored diversity, several important ones have not yet been evaluated and conserved.

Appemidi a unique aromatic pickle types mainly used for whole fruited tender mango pickles. These indigenous types are gaining importance in the export market because of their suitability for pickling as whole fruit (Radhakrishna Holla, 2007). But the local people are unaware of the importance of these indigenous types, plants were not managed scientifically and blatantly cutting the branches for harvesting of fruits to sell in local markets. Therefore a survey was conducted in this area to collect for conserving these unique types and also attempt was made to evaluate these types.

\section{Materials and Methods}

Germplasm with diverse genetic base is the major source for breeding programmes. Further, preservation of germplasm is a worldwide concern and conservation of specific diverse gene pools will be useful to breeders. Thus, an attempt was made to study the diversity of indigenous mango genotypes of Western Ghats regions of Chikmagalur District, Karnataka, by conducting a survey.

A three phase survey was conducted at Gonibeedu, Marebylu, Aldur, Balehonnur, Kudige, Soppinakadu, Arenuru, Magundi, Bynduru, Elelmadlu, Kanathi and Chimankudige places of Chikmagalur district, western ghat regions of Karnataka. Forty indigenous types were collected from different regions and each genotype names were given depending upon their place of collection. The leaves, Inflorescence, fruit and tree were characterized.

In each accession, three trees were selected to represent three replications and basic statistical measures such as mean, SEd, CV and SEm were worked outTen randomlyselected tender fruits (to the extent possible, before formation of the stonyendocarp) were used for biometric observations in each ccession and replication. Leaf characters like leaf shape, tip, leaf margin, texture, color of young flush, fragrance, leaf venation and Fruit characters like shape, flavour and latex flow were recorded. Weight of individual fruits was recorded and expressed in grams.

\section{Results and Discussion}

Various morphological characterizations were done for the 40 Appemidi genotypes collected from survey. In order to describe them systematically, morphological characterization of these entire 40 genotypes were carried out as per the standard descriptor for mango by IBPGR (2006) comprising of leaf, inflorescence, fruit and other parameters.

The application of morphological markers is the simplest of formal standardised methods of evaluating crop genetic diversity. Morphological characterisation is the first step that should be done before more profound biochemical or molecular studies are carried out (Hoogendijk and Williams, 2001) and it allows for the study of plant variation using visual attributes. The tree growth observed were erect, spreading and drooping in habit (Simi, 2006).

\section{Leaf characterization}

Leaf characters described include orientation of leaf shape, tip, leaf margin, texture, color of young flush, fragrance, leaf venation etc. leaf characters showed much variation among the genotypes. Naik and Gangolly(1950) also used leaf characters as secondary and tertiary characters for varietal identification.

The surveyed genotypes showed horizontal 
and semi erect type of leaf orientation with medium venation and coriaceous texture. Leaf shape predominantly oblong type, with wavy margins.

Majority of genotypes showed light green color of young leaves with dark green mature or fully developed leaves with mild fragrance (Table 1). Simi (2006) reported that lanceolate type of leaf shape and acuminate character of leaf tip and light green with brown tinge colour of young flush predominantly appeared in traditional mango types of southern Kerala.

\section{Inflorescence characterization}

The inflorescence characters predominantly noticed were the terminal position, pyramidal shape of inflorescence, light green colour and densely flowered panicles (Table 2). Simi (2006) also reported dominance of terminal inflorescences, pyramidal shape of inflorescence and densely flowered panicles in southern Kerala.

Jyothi et al., (2009) reported morethan 70 per cent of the mango tree shad broadly pyramidal inflorescence and are densely flowered which give them a bunch like appearance.

\section{Fruit characterization}

Fruits have been the major descriptors for identification of different varieties of fruit crops (Toili et al., 2013). Variability in mango types show that fruit shape was the most important and stable character for discriminating varieties from each other. Other fruit characters also have a degree of varying importance for the purpose of identification.

Presences of beak, fruit size, sinus, cavity of stalk insertion are also important for studying variability in mango germplasm (Ram and Rajan, 2003). Fruits have been exclusively used as the major descriptors for identification of different varieties of fruit crops (Toili et al., 2013).

Different fruit shapes were observed, i.e. round, elliptic, Obovoid, ovoid and oblong. Simi,(2006) also reported four types of fruit shapes such as round, oblong, ellipsoid, and oblong ellipsoid. NavPrem, (2014) characterized nine sucking mango genotypes and observed fruit shapes such as ovate, oblong and ovate oblong in their study.

The fruit length was ranged from $3.40 \mathrm{~cm}$ to $5.65 \mathrm{~cm}$ and the maximum fruit length was recorded in genotype Chimankudige-3 (5.65 $\mathrm{cm})$.Dinesh (2015) evaluated 130 mango varieties for fruit characteristics and reported that Maharaja Pasand, Sora and Tenneru had higher fruit length $(>20 \mathrm{~cm})$ (Table 3$)$.

The fruit diameter was differed significantly with a mean of $3.00 \mathrm{~cm}$ while, the fruit diameter was ranged from $2.22 \mathrm{~cm}$ to $3.92 \mathrm{~cm}$ among the cultivars studied. Maximum fruit diameter was recorded in genotype Arenuru-1 $(3.92 \mathrm{~cm}$. The mean fruit thickness was 2.33 $\mathrm{cm}$, while it ranged from $1.52 \mathrm{~cm}$ to $2.95 \mathrm{~cm}$. The maximum fruit thickness was recorded in genotype Agrahara $(2.95 \mathrm{~cm})$

The fruit weight of mango cultivars studied in the present investigation was ranged from $12.22 \mathrm{~g}$ to $21.12 \mathrm{~g}$ with a mean fruit weight of $16.33 \mathrm{~g}$, significantly maximum fruit weight was recorded in genotype Arenuru-2 (21.12 g) followed by Soppinakadu-2 (21.09g) (Table $3)$.

Vasugi et al., (2008), evaluated the unique pickle types and reported that fruit weight ranged from $17.43 \mathrm{~g}$ in Kanappe-1 to $191.75 \mathrm{~g}$ in Gaddalalli Appe which is attributed to the inherent nature of different accessions and are heritable under all environments. 
Table.1 Leaf Characteristics of forty surveyed Appemidi genotypes from Western Ghats regions of Chikmagalur

\begin{tabular}{|c|c|c|c|c|c|c|c|c|c|c|c|c|c|}
\hline Cultivar & $\begin{array}{l}\text { Leaf } \\
\text { attitude in } \\
\text { relation to } \\
\text { branch }\end{array}$ & $\begin{array}{l}\text { Thickness } \\
\text { of pelvinus }\end{array}$ & $\begin{array}{l}\text { Leaf } \\
\text { venation }\end{array}$ & Leaf texture & Leaf shape & $\begin{array}{l}\text { Leaf } \\
\text { margin }\end{array}$ & $\begin{array}{l}\text { Leaf } \\
\text { pubescence }\end{array}$ & $\begin{array}{l}\text { Colour of } \\
\text { young leaf }\end{array}$ & $\begin{array}{l}\text { Intensity of } \\
\text { anthocyanin } \\
\text { pigmentation }\end{array}$ & $\begin{array}{l}\text { Colour of } \\
\text { fully } \\
\text { developed leaf }\end{array}$ & $\begin{array}{l}\text { Leafy } \\
\text { bracts }\end{array}$ & Leaf tip & $\begin{array}{l}\text { Leaf } \\
\text { fragrance }\end{array}$ \\
\hline Agrahara & Horizontal & $\mathrm{T}$ & Medium & Coriaceous & Elliptic & Wavy & Present & LG & Low & DG & Present & Pointed & Strong \\
\hline Gonibeedu-1 & Horizontal & $\mathrm{T}$ & Medium & Coriaceous & Oblong & Wavy & Present & LB & High & DG & Present & Pointed & Strong \\
\hline Gonibeedu 2 & Horizontal & $\mathrm{T}$ & Medium & Coriaceous & Oblong & Wavy & Present & LB & High & DG & Present & Pointed & Strong \\
\hline Gonibeedu -3 & Semi errect & $\mathrm{T}$ & Medium & Coriaceous & Oblong & Wavy & Present & LB & Medium & DG & Present & Not Pointed & Strong \\
\hline Gonibeedu-4 & Semi errect & $\mathrm{T}$ & Medium & Coriaceous & Oblong & Wavy & Present & LB & Medium & DG & Present & Not Pointed & Strong \\
\hline Gonibeedu-5 & Semi errect & $\mathrm{T}$ & Medium & Coriaceous & Oblong & Wavy & Present & LG & Low & Green & Absent & Pointed & Mild \\
\hline Kamanmori-1 & Horizontal & $\mathrm{T}$ & Medium & Coriaceous & Oblong & Wavy & Present & LB & Medium & DG & Present & Pointed & Mild \\
\hline Kamanmori-2 & Semi errect & $\mathrm{T}$ & Medium & Coriaceous & Oblong & Wavy & Present & LG & Low & DG & Present & Pointed & Mild \\
\hline Chimankodige 1 & Semi errect & $\mathrm{T}$ & Medium & Coriaceous & Oblong & Wavy & Present & LG & Low & DG & Present & Pointed & Mild \\
\hline Chimankodige 2 & Semi errect & $\mathrm{T}$ & Medium & Coriaceous & Oblong & Wavy & Present & LG & High & Green & Absent & Pointed & mild \\
\hline Chimankodige-3 & Horizontal & $\mathrm{T}$ & Medium & Coriaceous & Oblong & Wavy & Present & Brown & High & DG & Present & Not Pointed & Strong \\
\hline Chimankodige-4 & I Semi errect & $\mathrm{T}$ & Medium & Coriaceous & Oblong & Wavy & Present & LG & Low & Green & Absent & Pointed & Mild \\
\hline Heruru-1 & Horizontal & $\mathrm{T}$ & Medium & Coriaceous & Oblongceolate & Wavy & Present & LG & Low & DG & Absent & Not Pointed & mild \\
\hline Heruru-2 & Horizontal & $\mathrm{T}$ & Medium & Coriaceous & Oblongceolate & Wavy & Present & LG & Low & DG & Absent & Not Pointed & mild \\
\hline Elemadlu-1 & Semi errect & $\mathrm{T}$ & Medium & Coriaceous & Oblong & Wavy & Present & LG & Low & DG & Present & Pointed & Mild \\
\hline Elemadlu-2 & Semi errect & $\mathrm{T}$ & Medium & Coriaceous & Oblong & Wavy & Present & LG & High & Green & Absent & Pointed & mild \\
\hline Marebilu-1 & Horizontal & $\mathrm{T}$ & Medium & Coriaceous & Oblongceolate & Wavy & Present & LG & Low & DG & Absent & Not Pointed & mild \\
\hline Marebilu-2 & Horizontal & $\mathrm{T}$ & Medium & Coriaceous & Oblongceolate & Wavy & Present & LG & Low & DG & Absent & Not Pointed & mild \\
\hline Marebilu-3 & Horizontal & $\mathrm{T}$ & Medium & Coriaceous & Oblong & Wavy & Present & Brown & High & DG & Present & Not Pointed & Strong \\
\hline Marebilu-4 & Semi errect & $\mathrm{T}$ & Medium & Coriaceous & Oblong & Wavy & Present & LG & Low & Green & Absent & Pointed & Mild \\
\hline Soppinakadu-1 & Semi errect & $\mathrm{T}$ & Medium & Coriaceous & Oblong & Wavy & Present & LG & Low & DG & Present & Pointed & Mild \\
\hline Soppinakadu-2 & Horizontal & $\mathrm{T}$ & Medium & Coriaceous & Oblongceolate & Wavy & Present & LG & Low & DG & Absent & Not Pointed & mild \\
\hline Soppinakadu-3 & Horizontal & $\mathrm{T}$ & Medium & Coriaceous & Oblongceolate & Wavy & Present & LG & Low & DG & Absent & Not Pointed & mild \\
\hline Kumrumane1 & Semi errect & $\mathrm{T}$ & Medium & Coriaceous & Oblong & Wavy & Present & LG & High & Green & Absent & Pointed & mild \\
\hline Kumrumane-2 & Horizontal & $\mathrm{T}$ & Medium & Coriaceous & Oblongceolate & Wavy & Present & LG & Low & DG & Absent & Not Pointed & mild \\
\hline Marigundi & Horizontal & $\mathrm{T}$ & Medium & Coriaceous & Oblong & Wavy & Present & Brown & High & DG & Present & Not Pointed & Strong \\
\hline Doobla & Horizontal & $\mathrm{T}$ & Medium & Coriaceous & Oblong & Wavy & Present & Brown & High & DG & Present & Not Pointed & Strong \\
\hline Kudige & Semi errect & $\mathrm{T}$ & Medium & Coriaceous & Oblong & Wavy & Present & LG & Low & Green & Absent & Pointed & Mild \\
\hline Bynduru & Semi errect & $\mathrm{T}$ & Medium & Coriaceous & Oblong & Wavy & Present & LB & Medium & DG & Present & Not Pointed & Strong \\
\hline Arenuru-1 & Horizontal & $\mathrm{T}$ & Medium & Coriaceous & Oblong & Wavy & Present & LB & High & DG & Present & Pointed & Strong \\
\hline Arenuru-2 & Horizontal & $\mathrm{T}$ & Medium & Coriaceous & Oblong & Wavy & Present & LB & Medium & DG & Present & Pointed & Mild \\
\hline
\end{tabular}




\begin{tabular}{|c|c|c|c|c|c|c|c|c|c|c|c|c|c|}
\hline Maagodu & Semi errect & $\mathrm{T}$ & Medium & Coriaceous & Oblong & Wavy & Present & LG & Low & DG & Present & Pointed & Mild \\
\hline Horatti jeerige- 1 & I Semi errect & $\mathrm{T}$ & Medium & Coriaceous & Oblong & Wavy & Present & LG & High & Green & Absent & Pointed & mild \\
\hline Horatti jeerige- 2 & 2 Horizontal & $\mathrm{T}$ & Medium & Coriaceous & Oblongceolate & Wavy & Present & LG & Low & DG & Absent & Not Pointed & mild \\
\hline Banakal & Horizontal & $\mathrm{T}$ & Medium & Coriaceous & Oblongceolate & Wavy & Present & LG & Low & DG & Absent & Not Pointed & mild \\
\hline Heggudlu & Semi errect & $\mathrm{T}$ & Medium & Coriaceous & Oblong & Wavy & Present & LG & Low & DG & Present & Pointed & Mild \\
\hline Kanathi-1 & Horizontal & $\mathrm{T}$ & Medium & Coriaceous & Oblong & Wavy & Present & Brown & High & DG & Present & Not Pointed & Strong \\
\hline Kanathi-2 & Semi errect & $\mathrm{T}$ & Medium & Coriaceous & Oblong & Wavy & Present & LG & Low & Green & Absent & Pointed & Mild \\
\hline Kanathi-3 & Semi errect & $\mathrm{T}$ & Medium & Coriaceous & Oblong & Wavy & Present & LB & Medium & DG & Present & Not Pointed & Strong \\
\hline Shantipura & Semi errect & $\mathrm{T}$ & Medium & Coriaceous & Oblong & Wavy & Present & LG & High & Green & Absent & Pointed & mild \\
\hline
\end{tabular}

Table.2 Inflorescence characteristics of forty surveyed Appemidi genotypes from Western Ghats regions of Chikmagalur

\begin{tabular}{|c|c|c|c|c|c|c|c|c|c|c|c|}
\hline Cultivar & $\begin{array}{l}\text { Regularity } \\
\text { of } \\
\text { flowering }\end{array}$ & $\begin{array}{l}\text { Inflorescenc } \\
\text { e position }\end{array}$ & $\begin{array}{l}\text { Inflorescence axis } \\
\text { growth habit }\end{array}$ & $\begin{array}{l}\text { Inflorescence } \\
\text { shape }\end{array}$ & $\begin{array}{l}\text { Inflorescence } \\
\text { anthocyanin } \\
\text { coloration }\end{array}$ & $\begin{array}{l}\text { Presence } \\
\text { of leafy } \\
\text { bracts }\end{array}$ & $\begin{array}{l}\text { Density of } \\
\text { flowers in } \\
\text { inflorescence }\end{array}$ & $\begin{array}{l}\text { Flower } \\
\text { type }\end{array}$ & $\begin{array}{l}\text { Inflorescence } \\
\text { colour }\end{array}$ & $\begin{array}{l}\text { Nature of } \\
\text { Disc }\end{array}$ & Hairiness \\
\hline Agrahara & Regular & Terminal & Semi Errect & Conical & weak & Absent & Dense & Penta & LG & Swollen & Absent \\
\hline Gonibeedu-1 & Regular & Terminal & Semi Errect & Pyiramidal & Medium & Absent & Sparse & Penta & YG & Swollen & Absent \\
\hline Gonibeedu 2 & Regular & Terminal & Semi Errect & Pyiramidal & Medium & Absent & Sparse & Penta & YG & Swollen & Absent \\
\hline Gonibeedu -3 & Biennual & Terminal & Semi Errect & Broadly pyiramidal & Medium & Absent & Dense & Penta & GwR & Swollen & Sparse \\
\hline Gonibeedu-4 & Biennual & Terminal & Semi Errect & Broadly pyiramidal & Medium & Absent & Dense & Penta & GwR & Swollen & Sparse \\
\hline Gonibeedu-5 & Biennual & Terminal & Semi Errect & Pyiramidal & Absent & Absent & Sparse & Penta & LG & Swollen & Sparse \\
\hline Kamanmori-1 & Biennual & Terminal & Semi Errect & Broadly pyiramidal & Absent & Absent & Sparse & Penta & YG & Swollen & Sparse \\
\hline Kamanmori-2 & Biennual & Terminal & Semi Errect & Conical & Absent & Absent & Sparse & Penta & YG & Swollen & Sparse \\
\hline Chimankodige 1 & Biennual & Terminal & Semi Errect & Conical & Absent & Absent & Sparse & Penta & YG & Swollen & Sparse \\
\hline Chimankodige 2 & Biennual & Terminal & Semi Errect & Conical & Strong & Absent & Dense & Penta & GwR & Swollen & Dense \\
\hline Chimankodige-3 & Biennual & Terminal & Semi Errect & Broadly pyiramidal & Strong & Absent & Sparse & Penta & GwR & Swollen & Sparse \\
\hline Chimankodige-4 & Biennual & Terminal & Semi Errect & Pyiramidal & Absent & Absent & Sparse & Penta & LG & Swollen & Sparse \\
\hline Heruru-1 & Biennual & Terminal & Semi Errect & Conical & Medium & Absent & Dense & Penta & GwR & Swollen & Absent \\
\hline Heruru-2 & Biennual & Terminal & Semi Errect & Conical & Medium & Absent & Dense & Penta & GwR & Swollen & Sparse \\
\hline Elemadlu-1 & Biennual & Terminal & Semi Errect & Conical & Absent & Absent & Sparse & Penta & YG & Swollen & Sparse \\
\hline Elemadlu-2 & Biennual & Terminal & Semi Errect & Conical & Strong & Absent & Dense & Penta & GwR & Swollen & Dense \\
\hline Marebilu-1 & Biennual & Terminal & Semi Errect & Conical & Medium & Absent & Dense & Penta & GwR & Swollen & Absent \\
\hline Marebilu-2 & Biennual & Terminal & Semi Errect & Conical & Medium & Absent & Dense & Penta & GwR & Swollen & Sparse \\
\hline Marebilu-3 & Biennual & Terminal & Semi Errect & Broadly pyiramidal & Strong & Absent & Sparse & Penta & GwR & Swollen & Sparse \\
\hline Marebilu-4 & Biennual & Terminal & Semi Errect & Pyiramidal & Absent & Absent & Sparse & Penta & LG & Swollen & Sparse \\
\hline Soppinakadu-1 & Biennual & Terminal & Semi Errect & Conical & Absent & Absent & Sparse & Penta & YG & Swollen & Sparse \\
\hline Soppinakadu-2 & Biennual & Terminal & Semi Errect & Conical & Medium & Absent & Dense & Penta & GwR & Swollen & Sparse \\
\hline Soppinakadu-3 & Biennual & Terminal & Semi Errect & Conical & Medium & Absent & Dense & Penta & GwR & Swollen & Absent \\
\hline
\end{tabular}


Int.J.Curr.Microbiol.App.Sci (2018) 7(7): 1010-1018

\begin{tabular}{|c|c|c|c|c|c|c|c|c|c|c|c|}
\hline Kumrumane1 & Biennual & Terminal & Semi Errect & Conical & Strong & Absent & Dense & Penta & GwR & Swollen & Dense \\
\hline Kumrumane-2 & Biennual & Terminal & Semi Errect & Conical & Medium & Absent & Dense & Penta & GwR & Swollen & Absent \\
\hline Marigundi & Biennual & Terminal & Semi Errect & Broadly pyiramidal & Strong & Absent & Sparse & Penta & GwR & Swollen & Sparse \\
\hline Doobla & Biennual & Terminal & Semi Errect & Broadly pyiramidal & Strong & Absent & Sparse & Penta & GwR & Swollen & Sparse \\
\hline Kudige & Biennual & Terminal & Semi Errect & Pyiramidal & Absent & Absent & Sparse & Penta & LG & Swollen & Sparse \\
\hline Bynduru & Biennual & Terminal & Semi Errect & Broadly pyiramidal & Medium & Absent & Dense & Penta & GwR & Swollen & Sparse \\
\hline Arenuru-1 & Regular & Terminal & Semi Errect & Pyiramidal & Medium & Absent & Sparse & Penta & YG & Swollen & Absen \\
\hline Arenuru-2 & Biennual & Terminal & Semi Errect & Broadly pyiramidal & Absent & Absent & Sparse & Penta & YG & Swollen & Sparse \\
\hline Maagodu & Biennual & Terminal & Semi Errect & Conical & Absent & Absent & Sparse & Penta & YG & Swollen & Sparse \\
\hline Horatti jeerige-1 & Biennual & Terminal & Semi Errect & Conical & Strong & Absent & Dense & Penta & GwR & Swollen & Dense \\
\hline Horatti jeerige-2 & Biennual & Terminal & Semi Errect & Conical & Medium & Absent & Dense & Penta & GwR & Swollen & Absen \\
\hline Banakal & Biennual & Terminal & Semi Errect & Conical & Medium & Absent & Dense & Penta & GwR & Swollen & Sparse \\
\hline Heggudlu & Biennual & Terminal & Semi Errect & Conical & Absent & Absent & Sparse & Penta & YG & Swollen & Sparse \\
\hline Kanathi-1 & Biennual & Terminal & Semi Errect & Broadly pyiramidal & Strong & Absent & Sparse & Penta & GwR & Swollen & Sparse \\
\hline Kanathi-2 & Biennual & Terminal & Semi Errect & Pyiramidal & Absent & Absent & Sparse & Penta & LG & Swollen & Sparse \\
\hline Kanathi-3 & Biennual & Terminal & Semi Errect & Broadly pyiramidal & Medium & Absent & Dense & Penta & GwR & Swollen & Sparse \\
\hline Shantipura & Biennual & Terminal & Semi Errect & Conical & Strong & Absent & Dense & Penta & GwR & Swollen & Dense \\
\hline
\end{tabular}


Table.3 Fruit morphological characterization of surveyed Appemidi genotypes

\begin{tabular}{|c|c|c|c|c|c|}
\hline Cultivars & Fruit Length $(\mathrm{cm})$ & $\begin{array}{l}\text { Fruit Diameter } \\
\text { (cm) }\end{array}$ & $\begin{array}{l}\text { Fruit thickness } \\
\text { (cm) }\end{array}$ & Fruit weight (g) & $\begin{array}{l}\text { Sap burning time } \\
\text { (seconds) }\end{array}$ \\
\hline Agrahara & 5.22 & 3.52 & 2.95 & 14.35 & 11.52 \\
\hline Gonibeedu-1 & 4.25 & 2.55 & 1.92 & 16.67 & 14.48 \\
\hline Gonibeedu 2 & 4.62 & 2.97 & 2.42 & 13.55 & 15.46 \\
\hline Gonibeedu -3 & 4.47 & 3.07 & 2.65 & 16.45 & 14.08 \\
\hline Gonibeedu-4 & 4.12 & 2.77 & 2.10 & 12.80 & 17.34 \\
\hline Gonibeedu-5 & 4.12 & 3.09 & 2.10 & 14.60 & 18.92 \\
\hline Kaman mori-1 & 5.23 & 2.98 & 2.18 & 13.35 & 16.98 \\
\hline Kaman mori-2 & 4.95 & 2.98 & 2.18 & 14.12 & 16.73 \\
\hline Chimankodige 1 & 4.15 & 3.48 & 2.25 & 13.22 & 16.55 \\
\hline Chimankodige 2 & 3.40 & 3.05 & 2.42 & 12.22 & 16.17 \\
\hline Chimankodige-3 & 5.65 & 2.95 & 1.52 & 15.75 & 16.15 \\
\hline Chimankodige-4 & 4.40 & 3.25 & 2.40 & 19.12 & 16.42 \\
\hline Heruru-1 & 4.50 & 2.22 & 2.59 & 18.32 & 16.32 \\
\hline Heruru-2 & 4.52 & 2.30 & 2.40 & 19.90 & 16.05 \\
\hline Elemadlu-1 & 5.23 & 2.55 & 2.60 & 17.30 & 16.58 \\
\hline Elemadlu-2 & 5.33 & 2.55 & 2.80 & 17.93 & 16.98 \\
\hline Marebilu-1 & 3.62 & 2.75 & 2.07 & 14.40 & 16.15 \\
\hline Marebilu-2 & 3.70 & 2.60 & 2.10 & 14.83 & 16.19 \\
\hline Marebilu-3 & 4.58 & 2.93 & 2.25 & 15.20 & 15.82 \\
\hline Marebilu-4 & 3.50 & 3.07 & 2.27 & 14.35 & 16.37 \\
\hline Soppinakadu-1 & 4.92 & 3.10 & 2.32 & 18.92 & 19.00 \\
\hline Soppinakadu-2 & 4.95 & 3.27 & 2.25 & 21.09 & 24.66 \\
\hline Soppinakadu-3 & 4.95 & 3.075 & 2.325 & 20.425 & 19.17 \\
\hline Kumrumane1 & 4.27 & 3.10 & 2.40 & 20.15 & 17.31 \\
\hline Kumrumane-2 & 4.27 & 3.10 & 2.37 & 20.00 & 17.51 \\
\hline Doobla & 4.60 & 2.70 & 2.15 & 16.19 & 25.95 \\
\hline Marigundi & 5.50 & 3.10 & 2.70 & 16.74 & 12.27 \\
\hline Kudige & 4.92 & 3.05 & 2.71 & 15.12 & 28.25 \\
\hline Bynduru & 4.16 & 2.75 & 2.07 & 14.30 & 12.31 \\
\hline Arenuru-1 & 3.80 & 3.92 & 2.07 & 15.31 & 27.02 \\
\hline Arenuru-2 & 4.42 & 3.38 & 2.25 & 21.12 & 27.88 \\
\hline Maagodu & 4.58 & 3.25 & 2.25 & 15.02 & 15.67 \\
\hline Horatti Jeerige-1 & 4.72 & 3.55 & 2.65 & 16.15 & 15.70 \\
\hline Horatti Jeerige-2 & 4.60 & 3.25 & 2.68 & 16.15 & 15.75 \\
\hline Banakal & 5.54 & 3.10 & 2.90 & 13.75 & 14.05 \\
\hline Heggudlu & 5.03 & 3.07 & 2.05 & 20.31 & 10.40 \\
\hline Kanathi-1 & 3.60 & 3.07 & 2.15 & 16.07 & 25.38 \\
\hline Kanathi-2 & 4.31 & 3.10 & 2.20 & 15.73 & 22.87 \\
\hline Kanathi-3 & 4.11 & 2.70 & 2.28 & 16.26 & 25.38 \\
\hline Shantipura & 4.53 & 2.78 & 2.35 & 16.09 & 15.05 \\
\hline Mean & 4.53 & 3.00 & 2.33 & 16.33 & 18.82 \\
\hline C.D. & 0.60 & 0.29 & 0.25 & 2.48 & -- \\
\hline SE (m) & 0.22 & 0.10 & 0.09 & 0.89 & 7.26 \\
\hline F-Test & $*$ & $*$ & $*$ & $*$ & $*$ \\
\hline C.V. & 9.51 & 6.82 & 7.76 & 10.84 & 77.32 \\
\hline
\end{tabular}


Sap burning is one of the traditional practices followed to select the best tender fruits for pickle purpose. Therefore we also carried out sap burning test for surveyed fruit samples. Out of forty genotypes the mean time was 18.82 seconds, the maximum sap burning time was recorded in the genotype Kudige (28.25 seconds).

These unique genotypes scions were collected and grafted to conserved our traditional diversity of mango. Conservation plays an important role to avoid the extinction of unique types and to maintain our traditional wealth.

In conclusion, knowledge of genetic variability strongly facilitates breeding for wider geographic adaptability. Several studies have been conducted from time to time on morphological description of mango (Burns and Prayag, 1921;Mukherjee, 1948; Naik and Gangolly, 1950; Singh and Singh,1956; Gangolly et al, 1957; Rajan et al, 1999 and Dinesh and Vasugi, 2002).

One of the characteristic features of mango varieties present in India has been expression of a character in a particular environment where the variety may have originated. In the present study, a given unique indigenous type of mango belonging to Western Ghat region has shown distinct characteristics. Conservation of indigenous types is a must to identify desirable traits in these genotypes for further use in the breeding programme. Evaluation of these types can help in the identification of better genotypes for products viz., pickle, which are gaining commercial importance.

\section{References}

Burns, W. and Prayag, S.H. 1921.Indian mangoes. J. RoyalHort. Soc., 26:755770 .
Gangolly, S.P., Singh, R., Katyal, S.L. and Singh, D. 1957.The book of the mango. Department of Agriculture, Bombay, Bull. 103.

Mukherjee, S.K. 1948. The varieties of mango (Mangifera indica L.) Bull. Bot. Soc. Bengal, 2:101-33.

Mukherjee, S.K. 1953. The mango its botany, cultivation, uses and future improvements, especially as observed in India. Econ. Bot., 7:130-162

Naik, K.C., and Gangolly, S.R. 1950.Classification and nomenclature of south Indian mangoes. The Madras Department of Agriculture, Super indent Printing Press, Madras, India

Radhakrishna Holla. 2007. Guna, Vaishistathegala Khajane Vanya Jaathiya maavu thaligagu Sujatha Sanchike,May 17-23.

Rajan, S., Negi, S.S. and Kumar, R. 1999.Catalogue of mango germplasm, Central Institute for Subtropical Horticulture, Lucknow, India.

Singh, L.B. and Singh, R.N. 1956.A monograph on the mangoes of Uttar Pradesh, Superintendent of Printing, UP Government, Lucknow.

IPGRI, 2006.Descriptors for Mango (Mangifera indica L.).International Plant Genetic Resources Institute, Rome, Italy.

Hoogendijk, M. And Williams, D. 2001.Characterizing the genetic diversity of homegarden crops: Some examples from Americas.2nd International Home Gardens Workshop, July 17-19, 2001, Witzenhausen, Federal Republic of Germany. Pp34-40.

Simi, S. 2006. Characterization of traditional mango (Mangifera indica L.) varieties of southern Kerala Part of Ph. D Thesis submitted to the Kerala Agricultural University, 171p.

Toili, M. E. M., Rimberia, F. K., Nyende, A. 
B., Mutwiwa, U., Kaluli, J. And Sila, D.2013. Assessing morphological diversity of mango germplasm from the upper Athi river (UAR) region of eastern Kenya. In: Scientific Conference Proceedings. 15th February 2013. pp 602-612.

Jyothi, M. L. Narayananakutty, M. C, Radha, T. And Puthummal, B. 2009. Pickling suitable mango in Kerala. National level training cum seminar on off seasonmango production. 7-8, December, 2009. Tamil Nadu Agricultural University, Coimbatore. pp.26-28.

Ram, S. And Rajan, S. 2003. Status Report on Genetic Resources of Mango in AsiaPacific Region, IPGRI Office for South Asia, New Delhi, India, pp. 6468.
Navprem, S., Gill, M.S. and Gill, P. P. S. 2014.Characterization of sucking type mango genotypes under sub-tropics of Punjab. Indian J. Hort. 71(1): 6-11.

Dinesh, M.R. and Vasugi, C. 2002. Catalogue of mangogermplasm, published by IIHR, Bangalore, pp.160.

Dinesh, M.R., Ravishankar, K.V., BhuwonSthapit, Parthasarathy, V.A., Sandya, B.S., Nischitha, P. And Lavanya, B. 2015, Genetic diversity in certain indigenous mango (Mangifera indica L.) varieties. Indian J. Plant Genet. Resour. 28(1): 153-160.

Vasugi C., Sekar K., Dinesh M. R. And Suresh E. R., 2008, Evaluation of unique mango accessions for wholefruit pickle, Journal of Horticultural Sciences. 3(2): 156- 160.

\section{How to cite this article:}

Veena, G.L. and Dinesh, M.R. 2018. Genetic Diversity in Appemidi (Unique Aromatic Pickle Mango) Genotypes from Western Ghats Regions of Chikmagalur (Karnataka), India. Int.J.Curr.Microbiol.App.Sci. 7(07): 1010-1018. doi: https://doi.org/10.20546/ijcmas.2018.707.122 